\title{
Velocity-Map Imaging for Emittance Characterization of Multiphoton Electron Emission from a Gold Surface
}

\author{
Hong Ye, ${ }^{1,2,3}$ Sebastian Trippel,,${ }^{1,3,}$ Michele Di Fraia, ${ }^{1,3, \dagger}$ Arya Fallahi, ${ }^{1}$ Oliver D. Mücke, ${ }^{1,3}$ \\ Franz X. Kärtner, ${ }^{1,2,3}$ and Jochen Küpper ${ }^{1,2,3}$ \\ ${ }^{1}$ Center for Free-Electron Laser Science, Deutsches Elektronen-Synchrotron DESY, \\ Notkestraße 85, 22607 Hamburg, Germany \\ ${ }^{2}$ Department of Physics, Universität Hamburg, Luruper Chaussee 149, 22761 Hamburg, Germany \\ ${ }^{3}$ The Hamburg Center for Ultrafast Imaging, Universität Hamburg, \\ Luruper Chaussee 149, 22761 Hamburg, Germany
}

(Received 19 July 2017; revised manuscript received 3 December 2017; published 12 April 2018)

\begin{abstract}
A velocity-map-imaging spectrometer is demonstrated to characterize the normalized emittance (rootmean-square, rms) of photoemitted electron bunches. Both the two-dimensional spatial distribution and the projected velocity distribution images of photoemitted electrons are recorded by the detection system and analyzed to obtain the normalized emittance (rms). With the presented distribution function of the electron photoemission angles, a mathematical method is implemented to reconstruct the three-dimensional velocity distribution. As a first example, multiphoton emission from a planar Au surface is studied via irradiation at a glancing angle by intense 45 -fs laser pulses at a central wavelength of $800 \mathrm{~nm}$. The reconstructed energy distribution agrees very well with the Berglund-Spicer theory of photoemission. The normalized emittance (rms) of the intrinsic electron bunch is characterized to be 128 and $14 \mathrm{~nm}$ rad in the $X$ and $Y$ directions, respectively. The demonstrated imaging spectrometer has the ability to characterize the normalized emittance (rms) in a few minutes with a fine energy resolution of $0.2 \mathrm{meV}$ in the image center and will, thereby, foster the further development of x-ray free-electron-laser injectors and ultrafast electron diffraction, and it opens up opportunities for studying correlated electron emission from surfaces and vacuum nanoelectronic devices.
\end{abstract}

DOI: 10.1103/PhysRevApplied.9.044018

\section{INTRODUCTION}

Time-resolved imaging of both transient molecular structure and condensed-phase dynamics with picometerfemtosecond spatiotemporal resolution has recently become possible with the advent of $\mathrm{x}$-ray free-electron lasers (XFELs) [1-8]. The high x-ray brilliance, coherence, and ultrashort pulse durations available from these sources are the key properties [9] that open up opportunities for new science. Therefore, precise control of the $\mathrm{x}$-ray pulse characteristics, including spectral coverage and temporal and spatial beam profiles are of utmost importance for advanced applications.

These parameters are directly influenced by the properties of the electron bunch generating the $\mathrm{x}$-ray pulses. Therefore, the accurate characterization of the electron-beam quality is indispensable for assessing available approaches in order to enable improvements of the underlying electron-beam technology. Because of its importance to the performance of accelerators, a multitude of techniques have been developed

\footnotetext{
*sebastian.trippel@cfel.de

Present address: Elettra-Sincrotrone Trieste S.C.p.A., 34149 Basovizza, Trieste, Italy.
}

in recent years to measure the transverse $[10,11]$ as well as the longitudinal energy spread. A detailed summary and the state of the art can be found elsewhere (see Ref. [12] and the references therein). In addition, high-quality electron bunches are instrumental in experiments where materials are studied via electron diffraction [13-15].

The key measure in electron-beam quality is electronbeam emittance, i.e., the transverse phase-space distribution of the generated electron bunches. To quantify electronbeam emittance as a function of photocathode composition and emission mechanism, we demonstrate a velocitymap-imaging (VMI) spectrometer that allows us to directly access the transverse velocity distribution (the term velocity refers to the vector quantity) of photoemitted electrons, enabling the measurement of normalized emittance (rootmean-square, rms) from various cathodes. Usually, emission mechanisms are classified as thermionic emission, photoemission, or tunneling emission under extraordinarily high electric fields. Recently, nanostructured and plasmonic photocathodes used with multiphoton or strong-field optical emission have been used as improved electron sources [16-23]. Both the experimental characterization and the theoretical description of the electron emittance from such 
cathodes is highly important, which serves as motivation for the direct VMI measurements developed here.

As a first proof-of-principle example, we report on quantitative measurements of multiphoton emission from a 400-nm-thick Au thin film at room temperature, which is excited with 45 -fs laser pulses centered at $800 \mathrm{~nm}$. Furthermore, these measurements allow us to benchmark the performance of this experimental setup. Quantumyield-dependent measurements are performed by recording the events of electrons impinging on the detector when varying the average laser power and the polarization angle, respectively. These experimental results confirm that fourphoton emission occurs from the planar Au surface. In our experiments, the two-dimensional (2D) transverse velocity or momentum distribution of photoemitted electrons is directly imaged onto the detector. An experimental threedimensional (3D) energy distribution is reconstructed from the measured 2D VMI data using a mathematical algorithm (vide infra) and compared to the theoretically derived 3D-space energy distribution from the Berglund-Spicer photoemission model [24-27]. The very good agreement of our experimental results with the theoretical model demonstrates the applicability of VMI to the characterization of the normalized emittance (rms) of photoelectron emitters.

\section{EXPERIMENTAL SETUP}

The velocity-mapping technique maps the velocity coordinates of particles onto a $2 \mathrm{D}$ detector without, to first order, the influence of the particles' spatial coordinates. To achieve this spatial-coordinates independence, a configuration of electrostatic lenses, in the simplest case using three parallel electrodes, is employed to spatially tailor the electric fields $[28,29]$. The setup can also be used to image and magnify the spatial coordinates while suppressing the effect of velocity coordinates, which is then referred to as spatial-map imaging (SMI) [28,30]. The spectrometer demonstrated here aims to characterize the electron normalized emittance ( $\mathrm{rms}$ ) via characterizing the average spread of electron coordinates in position-andmomentum phase space.

The schematic of the spectrometer is shown in Fig. 1. The sample is mounted on top of the sample holder, which can be retracted into a load-lock chamber. The load lock is designed for exchanging the sample without breaking the ultrahigh vacuum of the imaging system. When performing the electrostatic imaging experiments, the sample holder is transferred into the main chamber and brought in contact with the repeller plate to make sure they are at the same electric potential. The main chamber, maintained at $10^{-9}$ mbar, contains a stack of three cylindrically symmetric plates, labeled as repeller $(R)$, extractor $(E)$, and ground $(G)$ electrodes in Fig. 1. They are arranged in parallel, separated by $15 \mathrm{~mm}$, and, with applied potentials, serve as the electrostatic lens.

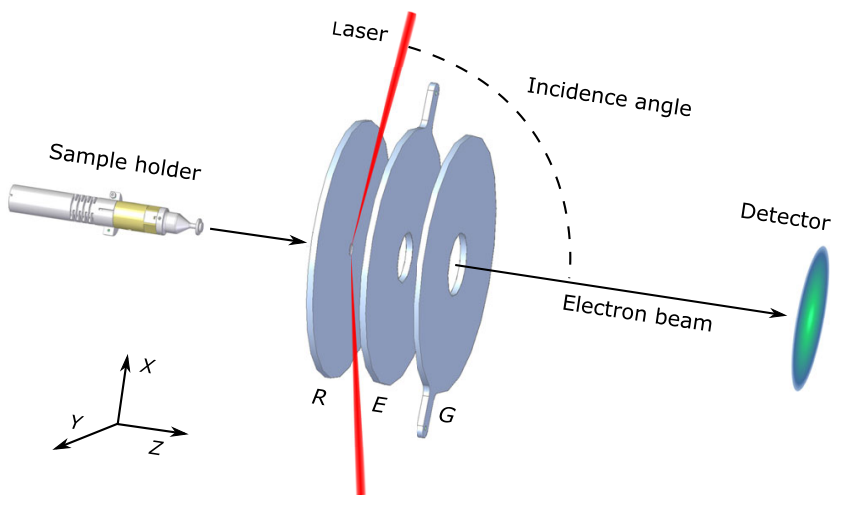

FIG. 1. Schematic of the VMI spectrometer consisting of three parallel electrodes: $R$, repeller; $E$, extractor; and $G$, ground. The sample is mounted on top of the holder, which can be retracted from this main chamber into a load-lock chamber.

This electrode configuration is followed by an approximately $0.5-\mathrm{m}$ drift tube, which ends with a detector assembly consisting of a double microchannel plate (MCP, Chevron configuration), a phosphor screen (P46) with a diameter of $40 \mathrm{~mm}$, and a CMOS camera (Optronis CL600 $\times 2$ ) for recording images of the electron distributions. The full configuration is shielded against stray fields by a mu-metal tube. An $800-n m, 45-f s$ Ti:sapphire laser amplifier with a $3-\mathrm{kHz}$ repetition rate is used to illuminate the sample at a glancing incidence angle of about $84^{\circ}$, with a laser focal intensity spot size of approximately $17 \times 160 \mu \mathrm{m}^{2} \mathrm{rms}$ on the sample. In our experiments, electron-distribution images are read out at a repetition rate of $1 \mathrm{kHz}$, limited by the camera-acquisition frame rate. The average number of electrons emitted per pulse is on the order of one or fewer, which excludes space charge effects that were reported previously [31].

To calibrate and optimize the spectrometer field configuration for both SMI and VMI, a fixed potential of $-6 \mathrm{kV}$ is applied to both the repeller plate and the sample holder while the ground plate is grounded; see Appendix A for details. While scanning the extractor voltage from -5.8 to $-4.3 \mathrm{kV}$, we observe the focusing of the electron bunch, depending on the extractor voltage [32]. This behavior is revealed by the rms of the electron-bunch size in the $X$ and $Y$ directions on the detector shown in Fig. 4.

SIMION [33] software is used to simulate the electric-field configuration and to calculate the electron trajectories from a 2D Gaussian source with $\sigma_{X}=140 \mu \mathrm{m}$ and $\sigma_{Y}=15 \mu \mathrm{m}$, yielding a rms curve that fits the experimental results. SMI is obtained at the minimum rms size, i.e., at an extractor voltage of $-5560 \mathrm{~V}$, corresponding to a magnification factor of 7.5. From the measured SMI data, the rms size is analyzed at $\sigma_{X}=158 \mu \mathrm{m}$ and $\sigma_{Y}=20 \mu \mathrm{m}$, which is in good agreement with the simulated electron-bunch size and the laser-focal-spot size. Importantly, in this experiment, hot spots due to sample surface roughness 
can conveniently be observed and located in SMI mode. Therefore, we are able to find suitably flat areas without hot spots within the laser-spot size, which can then be used for velocity mapping. The extractor voltage for VMI conditions is found at $-4790 \mathrm{~V}$ according to the SIMION simulations, and the calibration factor of velocity per pixel is $8014 \mathrm{~m} /(\mathrm{s}$ pixel $)$ on the detector. The details of the simulations and the experimental calibration are described in Appendix A.

In order to minimize field distortions, the sample front surface should be placed in the same plane as the repeller front surface. However, samples of different thickness lead to a position offset with reference to the repeller front, which strongly influences the field configuration. Therefore, the extractor voltage for operating in SMI and VMI mode is optimized by voltage adjustments of $[50,-50] \mathrm{V}$ and $[400,-200] \mathrm{V}$, respectively, to correct for a position offset of $[-0.5,0.5] \mathrm{mm}$. In this case, readjusting the potential right after exchanging a sample is necessary but quick (vide infra).

\section{EXPERIMENTAL RESULTS}

Figure 2(a) shows the photoemitted electron yield per laser shot as a function of incident laser peak intensity on a logarithmic scale. The error bars show the statistical errors of the photoemitted electron counts. The blue line reflects the results of a linear regression analysis that yields a slope of $c_{x} \approx 4.04$, with a coefficient of determination of $r^{2} \approx 0.997$.

The Fowler-DuBridge model for the $n$ th-order photoelectric current can be written, in a generalized form, as [34]

$$
J \propto A(1-R)^{n} I^{n} F\left(\frac{n h \nu-e \phi}{k T}\right),
$$

where $n$ is the number of photons, $h$ the Planck constant, $A$ the Richardson coefficient, $R$ the reflection coefficient from the metal surface, $I$ the incident light intensity, $\phi$ the metal work function, and $F(x)=\int_{0}^{\infty} \ln \left(1+e^{-(y+x)}\right) d y$ the Fowler function.

The experimental data in Fig. 2(a) follow a power law with a slope of about 4 , in agreement with a four-photon emission process, according to the nonlinear photoelectric effect, which indicates that the simultaneous absorption of four photons (photon energy $1.55 \mathrm{eV}$ at $800 \mathrm{~nm}$ ) has to take place to overcome the metal work function $W$ [35], which is reported as 5.31-5.47 eV for $\mathrm{Au}$ [36]. As shown in Fig. 2(b), varying the laser polarization angle, the photoemitted electron intensity reaches a maximum when the laser is $p$ polarized (the electric field is normal to the sample surface) and appears at minimum when it is $s$ polarized. For multiphoton emission at a certain incident light intensity, the electron yield depends mostly on the bulk absorption coefficient, expressed as the term $(1-R)^{n}$ in the Fowler-DuBridge model [35]. $R$ is calculated by
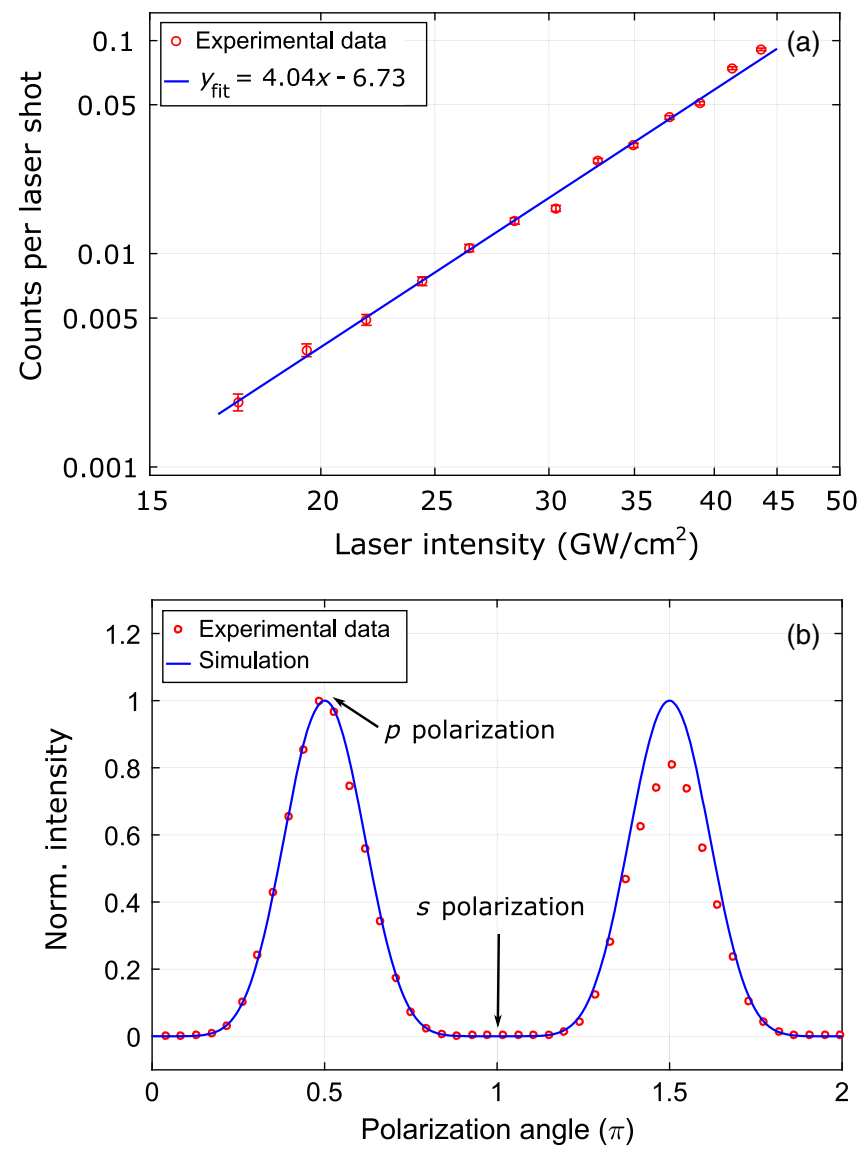

FIG. 2. Counts of the photoemitted electrons as functions of (a) the average laser power and (b) the laser polarization angle. The experimental data for polarization angles $>\pi$ are of reduced quality due to experimental instabilities, e.g., drifting laser pointing.

Fresnel equations with $n_{1}=1$ and $n_{2}=0.189+i 4.71$ [37] at an incidence angle of $84^{\circ}$. The plotted $(1-R)^{4}$ curve fits very well with the data, which again proves the fourth-order multiphoton process.

A velocity-map image from a planar Au surface is shown in the inset of Fig. 3(a). The image is integrated over $6 \times 10^{4}$ laser shots with an energy of approximately $50 \mathrm{~nJ}$, corresponding to a peak intensity of $4 \times 10^{10} \mathrm{~W} / \mathrm{cm}^{2}$ on the cathode. In laser-induced multiphoton emission, the emitted electron velocity vectors generally exhibit cylindrical symmetry along the direction normal to the sample surface. Therefore, the center of mass (c.m.) of the image is set as the coordinate origin. The corresponding angleintegrated radial velocity distribution of the projected electrons is plotted in Fig. 3(a) as a black line. To allow for comparison with the theoretical model, the $3 \mathrm{D}$ velocity or energy distribution is required.

Introducing a mathematical method similar to the onionpeeling algorithm [38], we are able to reconstruct the momentum or energy distribution when the angular distribution of emitted electrons is known. Fortunately, for 

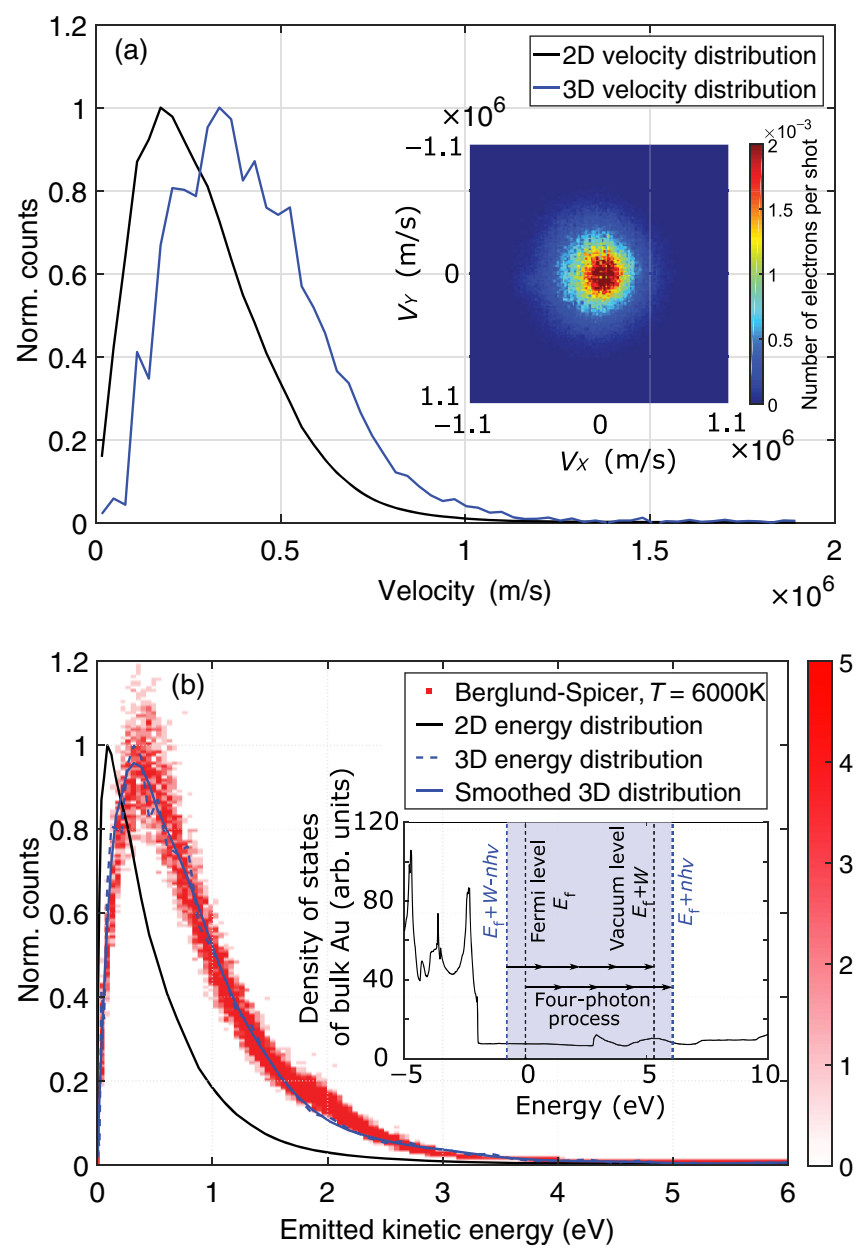

FIG. 3. (a) Projected 2D (black curve) and reconstructed 3D (blue curve) radial velocity distribution of the measured velocitymap image that is shown in the inset. (b) Reconstructed kineticenergy distribution and its simulation using the Berglund-Spicer model assuming an electron temperature of $6000 \mathrm{~K}$. The color bar of the $2 \mathrm{D}$ histogram represents the probabilities of photoelectron kinetic energies due to the photon-energy spectrum of the laser. (Inset) The density of states calculated for bulk Au, which is used in the Berglund-Spicer model simulation. The blue area depicts the four-photon-ionization range.

multiphoton emission, the intensity of photoemitted electrons at various angles $\theta$ can be derived from the BerglundSpicer model [25] as

$$
I(\theta) \propto \aleph^{2} \cos \theta \frac{1}{1+\alpha l(E)} \frac{1}{\sqrt{1-\aleph^{2} \sin ^{2} \theta}},
$$

where $\alpha$ is the optical absorption coefficient, $l(E)$ is the electron-electron scattering length for an electron of kinetic energy $E$, and $\boldsymbol{\aleph}$ expresses the electron analogy of refraction at the vacuum-metal boundary [39]. For a small $\boldsymbol{\aleph}$ (in our case, $\boldsymbol{\aleph}=0.275$ ), i.e., an incident photon energy $n h \nu$ comparable to the work function $W$, the equation can be simplified to $I(\theta) \propto \cos \theta[40,41]$. Therefore, the 3D velocity distribution can be reconstructed as is described in detail in Appendix B.

The reconstructed velocity distribution is plotted as a blue line in Fig. 3(a), and the smoothed energy distribution shown in Fig. 3(b). The energy distribution of the emitted electrons shows an energy spread of about $1 \mathrm{eV}$, which corresponds to the energy difference between a four-1.55-eV-photon excitation and the Au work function of $5.31 \mathrm{eV}$.

\section{DISCUSSION}

The Berglund-Spicer three-step model is employed as the analytic expression for the kinetic-energy distribution of the photoemitted electrons. As the model is derived for single-photon emission, it is implied in our analysis that the electrons at an initial energy state $E_{0}$ absorb a sufficient number of photons instantaneously, rather than sequentially, to be pumped to a higher energy state $E=E_{0}+n h \nu$. The kinetic-energy distribution for single-photon emission [24] is adapted to multiphoton emission as

$$
\begin{aligned}
N(E) d E \propto & \frac{K C(E) \alpha}{\alpha+1 / l(E)} d E \\
& \times\left[1+4\left(\frac{E-E_{f}}{n h \nu}-1+\ln \frac{n h \nu}{E-E_{f}}\right)\right],
\end{aligned}
$$

where $E_{f}$ is the Fermi energy of $\mathrm{Au}, C(E)=0.5 \times$ $(1-\sqrt{W / E})$ for $E \geq W$ is a semiclassical threshold function, and $l(E)$ is the electron-electron scattering length, which is proportional to $E^{-3 / 2}$. The absorption efficient $\alpha$ is calculated from the extinction coefficient $k=4.71$ as $\alpha=4 \pi k / \lambda$ and is taken as a constant $\alpha=7.7 \times 10^{5} \mathrm{~cm}^{-1}$ independent of the electron energy. $K$ is a correction factor related to both $C(E)$ and $\alpha l(E)$, which is between 0.5 and 1 .

To evaluate Eq. (3), the probability of a photon carrying the energy $h \nu$ is calculated from the measured laser spectrum in the range $760-850 \mathrm{~nm}$. To overcome the barrier of $5.31 \mathrm{eV}$, an electron is assumed to always absorb four photons $(n \equiv 4)$. The absorption of different photon energies leads to slight differences of the quantum yields at a certain emitted kinetic energy, as one can see from Fig. 3(b). The main consequence of absorbing photons with various energies is the intensity broadening, which is illustrated by the 2D histogram in Fig. 3(b). The temperature of the Fermi-Dirac distribution has been adjusted such that the mean of the histogram matches our experimental three-dimensional energy distribution. We mention that Eq. (3) includes only the emitted electrons that experience zero or one electron-electron scattering process during transport to the metal-vacuum surface. Electron-electron scattering is dominant over electron-phonon scattering and reshapes the energy distribution on a short timescale, i.e., during an ultrashort laser pulse.

The density of states (DOS), i.e., the number of states available for electrons at a certain energy level, is shown in 
the inset of Fig. 3(b). During the photoemission process, an energy state $E_{0}$ is first occupied by an electron, which is then excited to a higher energy state $E$, which is empty. As fermions, electrons obey the Pauli exclusion principle. In thermal equilibrium, the possibility of electrons occupying an available energy state is given by the Fermi-Dirac (FD) distribution $f_{\mathrm{FD}}$. However, excitation of a metal with ultrashort strong laser pulses initially creates a nonequilibrium distribution that then thermalizes via electronelectron scattering towards a Fermi-Dirac distribution. In gold, this thermalization occurs on a timescale of hundreds of femtoseconds $[42,43]$. Subsequently, the electrons cool down by dissipating energy into the lattice via electron-phonon scattering occurring on a longer, picosecond timescale.

In the following discussion, where we employ the Berglund-Spicer model in our analysis, we assume that the electronic system can be described by a Fermi-Dirac distribution with a quasiequilibrium electron temperature $T_{e}$. Hence, the appropriate densities of states and FD distributions are multiplied by the energy distribution as $N(E) d E f_{\mathrm{FD}}\left(E_{0}\right) \operatorname{DOS}\left(E_{0}\right)\left[1-f_{\mathrm{FD}}(E)\right] \operatorname{DOS}(E)$, resulting in the spectrum shown in Fig. 3(b).

The best fit with our reconstructed experimental energy distribution is obtained for an electron temperature of $6000 \mathrm{~K}$. This temperature is comparable to a previously observed electron temperature of $7000 \mathrm{~K}$ in surfaceenhanced multiphoton emission from copper [44]. The high-energy tail of the spectrum indicates that very "hot" electrons are photoemitted by the femtosecond laser pulse, which is consistent with the high excess energy deposited into the electronic system. For a tail up to $4 \mathrm{eV}$, above threshold photoemission-i.e., the absorption of one (or more) extra photons occurring together with the fourphoton process-might need to be taken into account [45]. Moreover, for our experimental conditions, we can neglect tunnel ionization, which could result in high-energy emitted electrons. Taking into account the Fresnel losses, we estimate the absorbed peak intensity for the recorded image, Fig. 3(a), to be approximately $4 \times 10^{9} \mathrm{~W} / \mathrm{cm}^{2}$. This intensity implies a Keldysh parameter $\gamma=\sqrt{W / 2 U_{p}} \approx$ $17 \gg 1$, which is well within the multiphoton emission regime; here, $U_{p} \propto \lambda^{2} I$ is the ponderomotive energy with laser wavelength $\lambda$ and intensity $I$.

Since both the measured quantum yield and the momentum distribution are in quantitative agreement with the Fowler-DuBridge and Berglund-Spicer models, as one would expect from multiphoton emission from a planar Au cathode, the VMI spectrometer is successfully implemented as a tool to characterize the photoemitted electrons from cathodes, especially for directly measuring the transverse momentum distribution. Assuming there is no correlation between the location of emission and the transverse momentum, the normalized emittance (rms) $\varepsilon_{n}$ is defined as [39]

$$
\varepsilon_{n_{\zeta}}=\frac{\sqrt{\left\langle\zeta^{2}\right\rangle\left\langle p_{\zeta}^{2}\right\rangle}}{m_{0} c}, \quad \text { with } \quad \zeta \in\{X, Y\},
$$

where $\left\langle\zeta^{2}\right\rangle$ is the spatial spread and $\left\langle p_{\zeta}^{2}\right\rangle$ is the momentum spread of the electron bunch. From the velocity-map image shown in the inset of Fig. 3(a), the normalized emittance (rms) of the planar Au photocathode irradiated by $45-\mathrm{fs}$, 800-nm laser pulses with a focal spot size of $\sigma_{X}=161 \mu \mathrm{m}$ and $\sigma_{Y}=17 \mu \mathrm{m}$ is characterized as $\varepsilon_{n_{X}}=128 \mathrm{~nm} \mathrm{rad}$ and $\varepsilon_{n_{Y}}=14 \mathrm{~nm}$ rad in the $X$ and $Y$ directions, respectively.

To decrease the intrinsic normalized emittance, one needs, in principle, to decrease either the emission area or the momentum spread. The former can be intuitively decreased by an extremely tight focal spot size or a sharp tip surface, which geometrically limits the emission area. To reduce the momentum or energy spread, choosing a proper material with an appropriate work function and irradiating it with a laser beam with matched photon energy, for example, the photoemission of $\mathrm{Cu}$ under 266-nm laser irradiation, is expected to help. A further reduction is expected when entering the strong-field emission regime, where the electrons are considered to adiabatically tunnel through the surface barrier with zero initial momentum and are then driven by the instantaneous optical field [16,46]. Under these conditions, electrons are expected to be emitted with a relatively small divergence angle and a significantly lower transverse momentum spread.

In order to characterize future low-emittance sources, high-resolution emittance measurements are mandatory. The presented spectrometer has that potential to measure the initial spatial and momentum distribution of the electrons - and, therefore, the emittance-in high resolution. The transverse energy resolution of the velocity mapping $d E=m v_{2 \mathrm{D}} d v_{2 \mathrm{D}}$ linearly increases with the transverse velocity $v_{2 \mathrm{D}}$ in a VMI spectrometer. In our case, the spatial resolution of the detector, the Chevron MCP, is $100 \mu \mathrm{m}$. This matches the resolution provided by a single camera pixel $\left[d v_{2 \mathrm{D}}=8014 \mathrm{~m} /(\mathrm{s}\right.$ pixel $\left.)\right]$. Therefore, the transverse energy resolution of the spectrometer is given by $0.2 \mathrm{meV} \leq d E \leq 90 \mathrm{meV}$. The lower boundary corresponds to the resolution in the detector center, whereas the upper boundary is the resolution at the edge. Therefore, compared to other techniques, our spectrometer has an unprecedented transverse energy resolution in the center.

For our current settings, the maximum detectable transverse energy is on the order of $10 \mathrm{eV}$. These settings result in a relative resolution of $<1 \%$ at the edge of the detector, again given by the resolution of the detector (or camera). It should be noted that the current transverse energy resolution could, in principle, simply be increased by using a larger detector, a longer drift region, and a higher-resolution camera. A 3 times better resolution of $0.07 \mathrm{meV} \leq d E \leq 30 \mathrm{meV}$ is obtainable, e.g., with a $12-\mathrm{cm}$-diameter detector, a $1.5-\mathrm{m}$ drift tube, and a high-resolution camera. The current spatial resolution in SMI mode is given by $100 \mu \mathrm{m} / 7.5=13 \mu \mathrm{m}$ on 
the cathode, which is sufficient to measure the initial distribution of the electrons. With the same changes on the setup as discussed above, a $3 \times$ better spatial resolution on the cathode can be reached. This improvement results in an overall resolution in the emittance given by $0.5 \mathrm{~nm} \mathrm{rad}$, with the possibility of improving it to about $0.06 \mathrm{~nm}$ rad for future experiments. A discussion of the systematic errors is provided in Appendix C, which shows that they have no significant impact on the resolution of the presented apparatus.

A comparison of existing methods to characterize an ultralow-emittance photocathode is presented in Ref. [12]. They all have in common that the apparatus and the corresponding transfer functions have to be modeled. The transverse energy resolution is typically worse than the one obtained here. The most outstanding advantage of the VMI spectrometer is that an entire single Newton sphere is captured at once, and various Newton spheres are simply superimposed. This implies that the mapping is nondestructive, in the sense that no filter functions like retarding voltages in combination with pinholes need to be applied as, e.g., for the 2D energy analyzer [47]. Therefore, the mapping avoids slow electrons and their extremely stray-field-sensitive trajectories.

Free expansion, reported as the simplest method by far [12], is the closest technique to the VMI spectroscopy demonstrated here. This technique is conceptually the analog to the early ion imaging experiments before the invention of the velocity-map-imaging spectrometer [28]. However, the commonly present electrode grids lead to transmission reduction, severe trajectory deflection, and blurring due to the non-point-source geometry. In addition, the incident laser in the demonstrated free expansion setup [48] is focused onto the sample through the grid, which seriously deforms the starting intensity distribution.

The high energy resolution of the VMI in comparison to the free expansion technique is attributed to the inhomogeneous electric field in the spectrometer. This field configuration allows us, in first order, to get rid of the spatial contribution in the velocity coordinates. Therefore, a single measurement is sufficient to obtain the velocity map without contributions from the initial source distribution. Furthermore, non-cylindrically-symmetricvelocity distributions, e.g., obtained from nanotips, can be measured as well. As a final touch, operating the VMI spectrometer under SMI conditions allows the mapping of the initial source distribution, which circumvents the modeling of the active laser-matter interaction area. Overall, the simplicity of the VMI spectrometer and the supershort measurement times, typically lasting only a few minutes, enables an easy integration into moresophisticated electron sources.

\section{CONCLUSIONS AND OUTLOOK}

In this paper, we demonstrate an electron spectrometer with VMI and SMI capabilities, which intuitively allows for high-resolution measurements of the normalized emittance (rms) of photocathodes through a direct observation of the transverse position and momentum distributions. We verify and benchmark the capabilities of the instrument in a proof-of-concept experiment in which we characterize the photoemitted electrons from a 400-nm thin Au film. For ultrashort femtosecond laser pulses with a peak intensity lower than $10^{12} \mathrm{~W} / \mathrm{cm}^{2}$ and a central wavelength of $800 \mathrm{~nm}$, which corresponds to $\gamma=1$, multiphoton emission is shown to be the dominant contribution to the entire electron current.

We intend to utilize this technique for the emittance characterization of electron bunches that are strong-field emitted from nanotips under optical field irradiation. Such devices should show superior emittance [17,20]. Moreover, the small radii of the sharp tips realize a field enhancement, which dramatically lowers the laser power required for entering the strong-field regime and thus avoids damaging of the cathodes. Our ongoing work aims at the characterization of electron emission from nanostructured array emitters, which are predicted to provide high-current, low-emittance coherent electron bunches in the strongfield-emission regime. The demonstrated imaging spectrometer will thereby foster the further development of XFELs and ultrafast electron microscopy and diffraction $[20,49]$ and also opens up opportunities in the study of correlated electron emission from surfaces [50] and of vacuum nanoelectronic devices [51].

\section{ACKNOWLEDGMENTS}

We gratefully acknowledge the helpful discussions with Jens S. Kienitz and Nele Müller, the expert technical support of Thomas Tilp, and Nicolas Tancogne-Dejean for providing the computed density-of-states data of bulk Au. Besides DESY, this work has been supported by the excellence cluster "The Hamburg Center for Ultrafast Imaging-Structure, Dynamics and Control of Matter at the Atomic Scale" (CUI, DFG-EXC1074), the priority program QUTIF (SPP1840 SOLSTICE) of the Deutsche Forschungsgemeinschaft, the European Research Council under the European Union's Seventh Framework Programme (FP7/2007-2013) through the Consolidator Grant COMOTION (ERC-Küpper-614507) and the Synergy Grant AXSIS (ERC-Kaertner-609920), the Helmholtz Association "Initiative and Networking Fund," and the accelerator on a chip (ACHIP) program funded by the Betty and Gordon Moore Foundation.

\section{APPENDIX A: SPECTROMETER CHARACTERIZATION}

The electron spectrometer is characterized experimentally, accompanied by simulations, in order to determine the focusing conditions for the SMI and VMI modes; see Fig. 1 for the experimental setup. Figure 4 shows the measured 


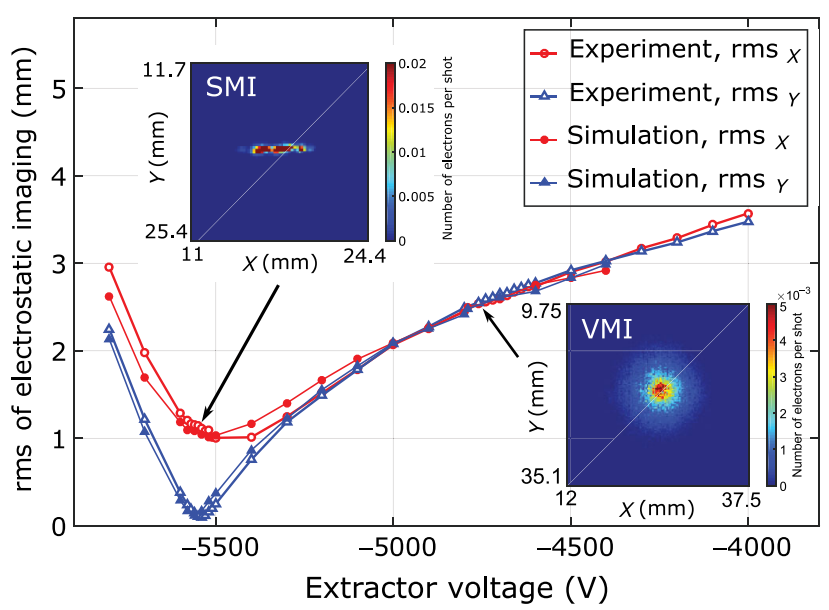

FIG. 4. Experimental (hollow) and simulated (solid) root-meansquare widths of electron spatial distributions on the 2D detector versus the focusing extractor voltage in both the $X$ and $Y$ directions. (Insets) SMI and VMI detector images for the indicated positions.

rms of the spatial electron distribution in the $X$ and $Y$ directions on the detector as a function of the extractor voltage, together with the corresponding results from SIMION [33] electric-field and particle trajectory simulations. A behavior similar to that in Ref. [32] is observed.

The strongest focusing of the electron bunch onto the detector is achieved at an extractor potential of $-5560 \mathrm{~V}$, which is thus identified as the SMI voltage. The rms at this voltage shows the magnified laser-surface-interaction area. The slightly different focusing behavior of the electron bunch in the $X$ and $Y$ directions is attributed to the asymmetric initial electron-bunch size due to the glancing incidence irradiation and the finite kinetic energy of the electrons.

When increasing the extractor voltage, the electron bunch diverges. Based on our simulations, the extractor voltage for VMI is approximately $-4790 \mathrm{~V}$. For a full calibration of the spectrometer, the simulations are used to study the field configuration and the electron trajectories in those fields for the given electrode configurations and the particles' initial distributions. In Fig. 4, the simulated rms of the electron bunch at the detector position is plotted as a function of the extractor voltage. The simulations are carried out for 2000 electrons from an initial spatial 2D Gaussian distribution for each simulated point. The c.m. of this distribution is given by $(X, Y)=(0,0)$ and a $Z$ coordinate matching the sample surface; the widths are $\sigma_{X}=140 \mu \mathrm{m}, \sigma_{Y}=15 \mu \mathrm{m}$, and $\sigma_{Z}=0$. The initial momentum distribution is given by a uniform half sphere with an uniform kinetic-energy distribution of electrons in the range of $[0.1,0.6] \mathrm{eV}$.

The c.m. of the electron distribution as a function of the initial starting position of the electrons-i.e., the laser focus position on the sample-is used to experimentally calibrate

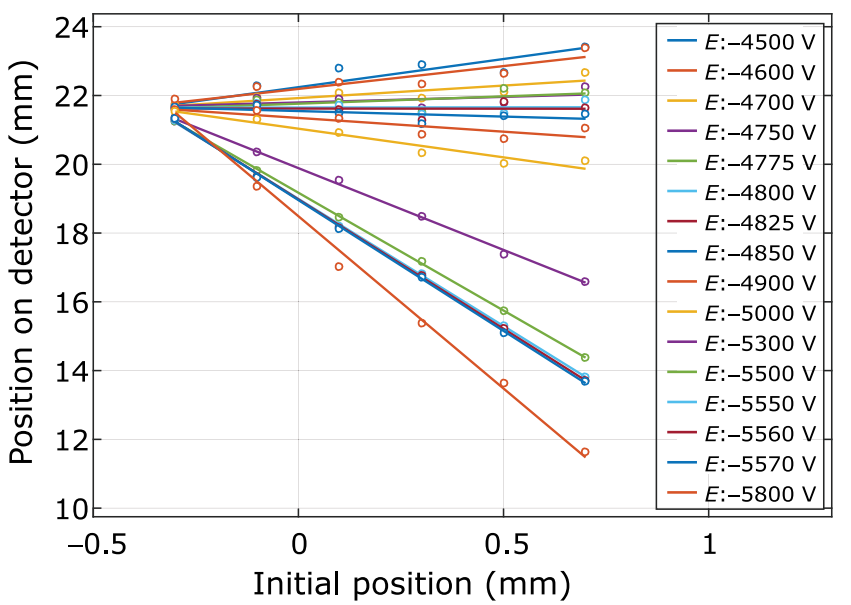

FIG. 5. Position dependence of the center of mass of the electron distribution on the detector on the initial source position for various extractor potentials ranging from -5800 to $-4500 \mathrm{~V}$.

the voltage for velocity-map imaging. Figure 5 shows the c.m. as a function of the laser position for various voltages together with straight-line fits. A decrease of the slope with decreasing extractor voltage is observed. Figure 6 depicts the slope of each measurement in Fig. 5 as a function of the extractor voltage together with a quadratic fit and the corresponding simulation results. The error bar for the experimental points is given by the first-order coefficient error of each fit with $95 \%$ confidence bounds. VMI mode is obtained at the zero crossing of this curve, i.e., at $-4790 \mathrm{~V}$, as, for this voltage, the distribution, to first order, becomes independent of the starting position. The data show good agreement with the simulations, confirming that the extractor voltage for operating the VMI is $-4790 \mathrm{~V}$. From the simulations, the imaging setup is calibrated regarding the

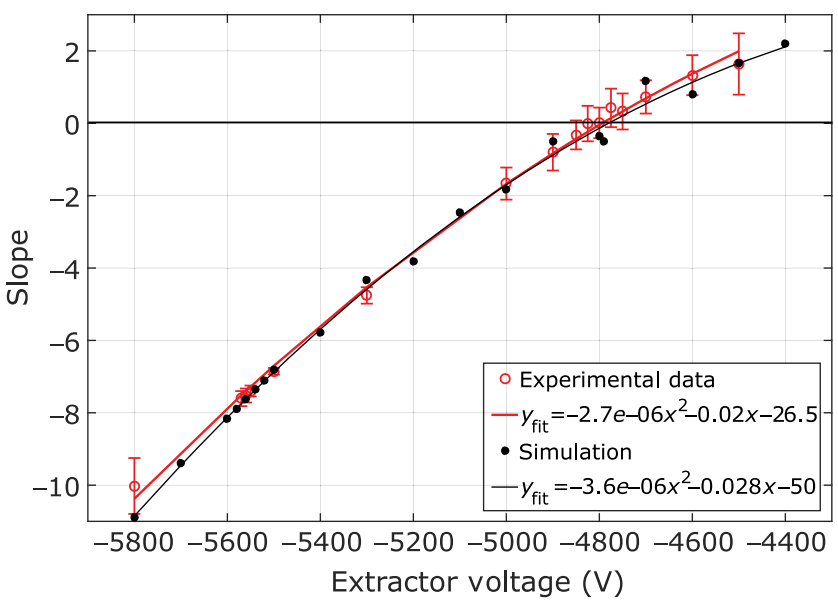

FIG. 6. Slope of the experimental laser position-dependent c.m. of the spatial distribution at the detector as a function of the extractor voltage (the red circles) with a quadratic fit (the red line). The black points and the black line indicate the corresponding simulated results. 
TABLE I. Voltages (in V) applied for operation in SMI and VMI mode.

\begin{tabular}{llccc}
\hline \hline & Repeller & Extractor & Ground & Sample \\
\hline SMI & -6000 & -5560 & 0 & -6000 \\
VMI & -6000 & -4790 & 0 & -6000 \\
\hline \hline
\end{tabular}

transverse electron velocities to $8014 \mathrm{~m} /(\mathrm{s}$ pixel) on the detector. The resulting voltages for operation in the SMI and VMI modes are listed in Table I.

Figure 7 shows the experimental and theoretical c.m.'s of the electron distribution at the detector as a function of the lens position, which is used to focus the laser beam onto the sample for SMI $\left(V_{E}=-5560 \mathrm{~V}\right)$. The straight lines are fits to the data. The difference in the slope between the $X$ and $Y$ directions is due to the glancing incidence angle $\theta$. The laser-spot position on the sample moves $1 / \cos \theta$ times farther in $X$ than in $Y$ when displacing the laser beam the same distance by a translation stage. For the $Y$ direction, we obtain a magnification factor of about 7.5 from the fit. For the $X$ direction, a slope of approximately 72.7 is obtained. The resulting ratio between the two slopes is 9.7, corresponding to an incident angle of $84^{\circ}$. The SIMION simulation results, also shown in Fig. 7, are in good agreement with the data.

The focusing conditions for the SMI and VMI modes depend strongly on the position of the sample inside the velocity-map-imaging spectrometer. Figure 8 shows the simulated extractor voltages necessary for the SMI and VMI modes for various sample displacements with respect to the front surface of the repeller plate. These simulations show that either the sample position has to be known —or at least reproduced - to a very high precision or calibration measurements have to be performed when a new sample is

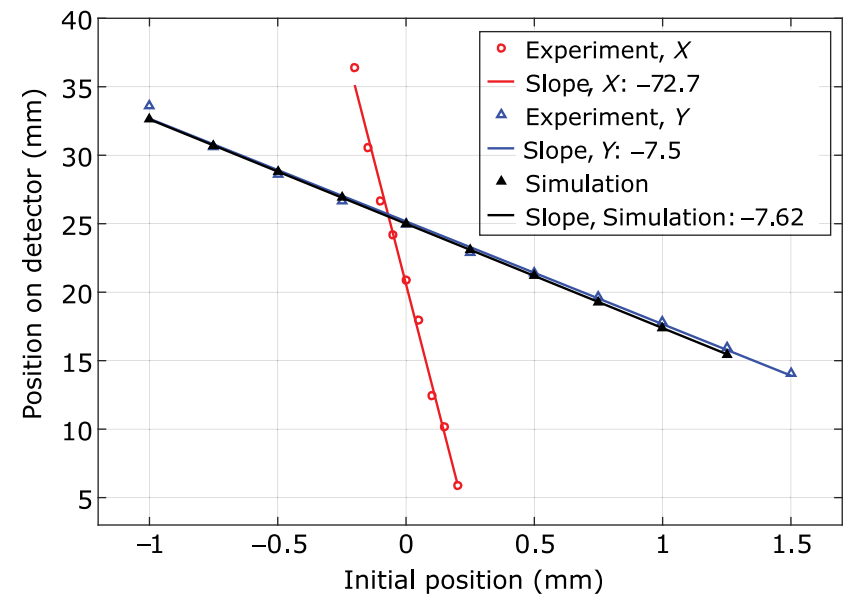

FIG. 7. The c.m. of electrostatic imaging on the detector as a function of the initial source position for SMI mode, i.e., an extractor voltage of $-5560 \mathrm{~V}$. The slope in the $Y$ direction is the spatial magnification factor. The ratio between the $X$ and $Y$ directions confirms the incidence angle of the laser beam of $84^{\circ}$.
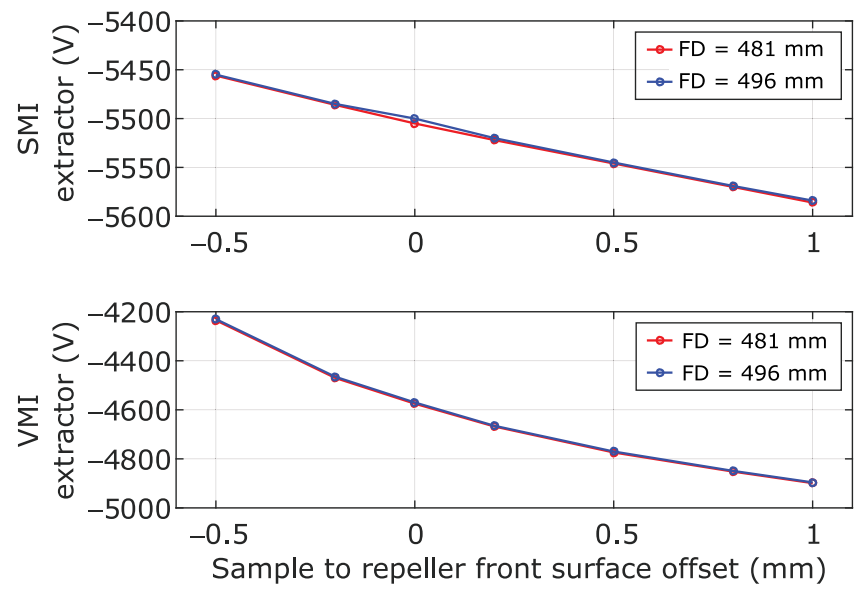

FIG. 8. (Top panel) SMI and (bottom panel) VMI extractor potential for different position offsets from the sample front to the repeller front surfaces. FD stands for flying distance in the figure legend.

inserted into the spectrometer. Fortunately, with the protocol described in our paper, this calibration can be done quickly. In addition, the dependence of the extractor voltage on the flight distance is investigated (the red points and lines). Our simulations show that this uncertainty is uncritical compared to the exact sample position in the spectrometer.

\section{APPENDIX B: RECONSTRUCTION ALGORITHM}

Our reconstruction algorithm for the conversion of the $2 \mathrm{D}$ projected velocity distribution to the $3 \mathrm{D}$ distribution is based on the assumption that the angular distribution of the photoemitted electrons is known. For our simulations, a cosine function $I(\theta) \propto \cos \theta[40,41]$, derived from the Berglund-Spicer model [25] as discussed in the main text, is applied in the algorithm. In addition, it is assumed that, for multiphoton emission, the angular distribution is independent of the modulus of the three-dimensional velocity vector. The $3 \mathrm{D}$ velocity distribution is then obtained from the 2D projected distribution by a matrix method similar to onion peeling [38]. For multiphoton emission from a planar $\mathrm{Au}$ surface, the electrons are assumed to be photoemitted within a half sphere of $\varphi \in[0,2 \pi], \theta \in[0, \pi / 2]$. The photoemitted electron distribution has cylindrical symmetry with respect to the surface normal of the sample.

Figure 9(a) shows a scatterplot for a single 3D velocity $v_{i}$ distribution given by $f(v, \theta)=\delta\left(v-v_{i}\right) \cos \theta$. Figure 9(b) shows the projection of this distribution onto the 2D detector surface. It can be derived that the projected velocity distribution for this special case is

$$
P_{i}\left(v_{x}, v_{y}\right)=\int f(v, \theta) d v_{z}=\left\{\begin{array}{ll}
C & \text { for } v_{x, y}<v_{i} \\
0 & \text { otherwise }
\end{array},\right.
$$



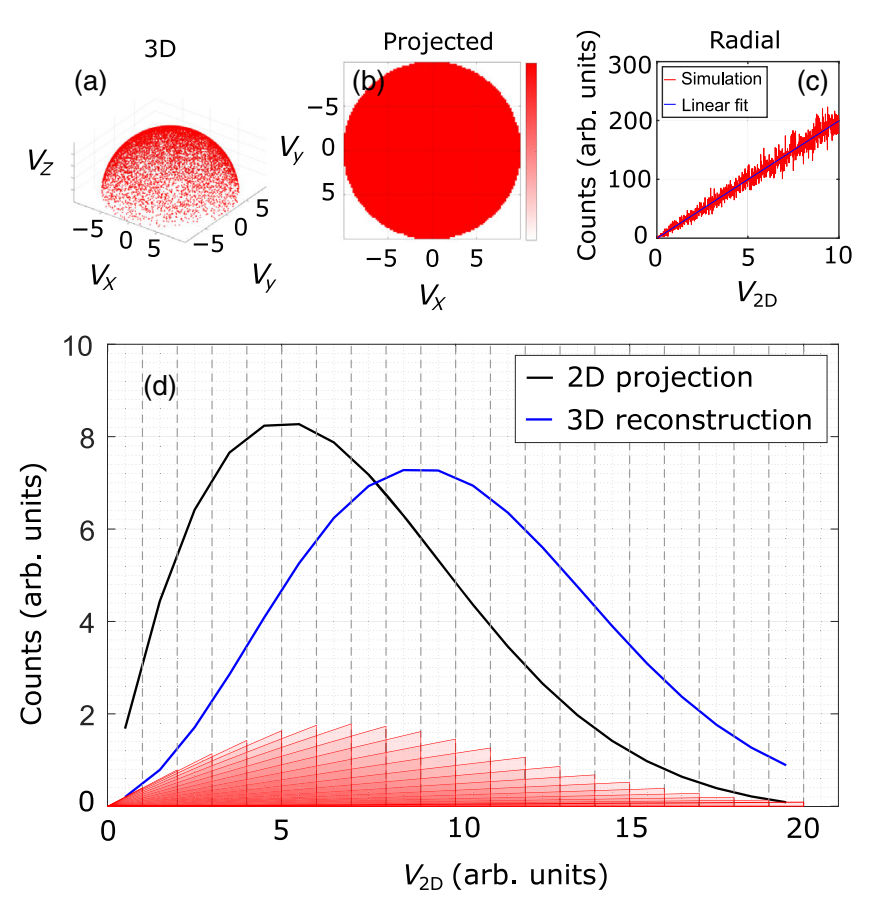

FIG. 9. (a)-(c) Representation of a simulated electron bunch with a single $3 \mathrm{D}$ velocity $v_{i}$ and an angular distribution of a cosine function. (a) In 3D, forming a spherical surface. (b) In 2D, yielding a uniform distribution in the detector plane. (c) In 1D, showing a linearly increasing radial velocity $v_{2 \mathrm{D}}$ with the distance from the distribution c.m. (d) A conceptual diagram of the reconstruction algorithm. The area of each red triangle at the bottom indicates the number of photoemitted electrons having the same $3 \mathrm{D}$ velocity. The corresponding distribution curve is plotted as a blue curve. The black curve is the $2 \mathrm{D}$ projection distribution curve, summing up the number of photoemitted electrons within each interval of the same transverse velocity. The gray dashed lines indicate the transverse velocity intervals used in this projection.

where $C$ is a constant. As shown in Fig. 9(b), the projected velocity distribution of $f(v, \theta)$ is constant inside the circular phase-space area of radius $v_{i}$. Furthermore, Fig. 9(c) shows the radial distribution obtained from the projected velocity distribution given by

$\rho_{i}\left(v_{2 \mathrm{D}}\right)=\int P_{i}\left(v_{x}, v_{y}\right) d \theta_{2 \mathrm{D}}=\left\{\begin{array}{ll}2 \pi C v_{2 \mathrm{D}} & \text { for } v_{2 \mathrm{D}}<v_{i} \\ 0 & \text { otherwise }\end{array}\right.$,

where $v_{2 \mathrm{D}}=\sqrt{v_{x}^{2}+v_{y}^{2}}$. In the reconstruction, each radial distribution $\rho_{i}\left(v_{2 \mathrm{D}}\right)$ is built up by a triangle as sketched in Fig. 9(d). $v_{i}$ is taken equally spaced and form the intervals confined by the neighboring gray dashed lines. The $2 \mathrm{D}$ projected distribution is related to the $3 \mathrm{D}$ distribution $f_{i}$ through a transfer matrix $\mathbf{M}$,

$$
\rho_{i}=\mathbf{M} f_{i}
$$

with $\mathbf{M}$ given by

$$
\mathbf{M}=\left(\begin{array}{ccccc}
1 & 1 / 4 & 1 / 9 & 1 / 16 & \ldots \\
0 & 3 / 4 & 3 / 9 & 3 / 16 & \ldots \\
0 & 0 & 5 / 9 & 5 / 16 & \ldots \\
0 & 0 & 0 & 7 / 16 & \ldots \\
\vdots & \vdots & \vdots & \vdots & \ddots
\end{array}\right) .
$$

The 3D distribution can finally be obtained by an inversion of the measured 2D-projected distribution,

$$
f_{i}=\mathbf{M}^{-1} \rho_{i} .
$$

\section{APPENDIX C: SYSTEMATIC ERRORS}

We further discuss systematic errors with respect to the resolution of the spectrometer. First, the power supplies (FUG HCP 14-12500) have an accuracy of $25 \mathrm{~V}$. This accuracy affects the voltages applied to both the repeller and extractor electrodes, and it results in a systematic error in the transverse energy. The radius on the detector corresponding to a transverse velocity $v_{2 \mathrm{D}}$ is given by $r=c t_{\mathrm{TOF}} v_{2 \mathrm{D}}$, where $t_{\mathrm{TOF}}$ denotes the time of flight (TOF) of the electrons. The magnification factor $c \approx 1$ of the spectrometer for velocity mapping is, fortunately, independent of the specific voltages applied to the spectrometer as long as the ratio of the repeller and extractor voltages, which is experimentally optimized to 1.253 , is fixed. Therefore, this factor can be simulated and calibrated precisely.

The error for the mapping is then given only by the TOF deviation due to the absolute repeller voltage bias. A voltage of $6000 \pm 25 \mathrm{~V}$ results in $t_{\mathrm{TOF}}=10.88 \pm 0.02 \mathrm{~ns}$. This value results in a maximum error on the edge of the detector of $40 \mu \mathrm{m}$, which is about a factor of 2 smaller than the resolution of the detector assembly. Thus, the systematic errors due to the accuracy of the power supply is negligible. For the same reason, the work functions for the materials used, typically on the order of a few electron volts, are negligible as well.

An additional contribution to the systematic error arises from the accuracy in determining the distance between the cathode plane and the detector. A 1-mm accuracy results in a change in the time of flight of about $20 \mathrm{ps}$, again corresponding to a maximum error on the edge of the detector of $40 \mu \mathrm{m}$, which is again small compared to the resolution of our detector. In addition, our simulations show that the largest error due to aberrations of the spectrometer in VMI mode is given by $100 \mu \mathrm{m}$ at the outermost part of the detector. This is again of the same order of magnitude as our resolution in this area.

It may be noted that adding up all errors degrades the resolution, but only in the very outer part of the image. 
In the image center, where most of the signal is detected, the systematic error is still below the resolution. Furthermore, aberrations of the spectrometer in SMI mode are negligible on the length scale corresponding to the laser-spot size on the cathode.

[1] H. N. Chapman et al., Femtosecond diffractive imaging with a soft-x-ray free-electron laser, Nat. Phys. 2, 839 (2006).

[2] A. Barty, S. Boutet, M. J. Bogan, S. Hau-Riege, S. Marchesini, K. Sokolowski-Tinten, N. Stojanovic, R. Tobey, H. Ehrke, A. Cavalleri, S. Düsterer, M. Frank, S. Bajt, B. W. Woods, M. M. Seibert, J. Hajdu, R. Treusch, and H. N. Chapman, Ultrafast single-shot diffraction imaging of nanoscale dynamics, Nat. Photonics 2, 415 (2008).

[3] L. Young et al., Femtosecond electronic response of atoms to ultra-intense x-rays, Nature (London) 466, 56 (2010).

[4] A. Barty et al., Self-terminating diffraction gates femtosecond $\mathrm{x}$-ray nanocrystallography measurements, Nat. Photonics 6, 35 (2012).

[5] B. Rudek et al., Ultra-efficient ionization of heavy atoms by intense x-ray free-electron laser pulses, Nat. Photonics 6, 858 (2012).

[6] A. Barty, J. Küpper, and H. N. Chapman, Molecular imaging using x-ray free-electron lasers, Annu. Rev. Phys. Chem. 64, 415 (2013).

[7] J. Küpper et al., X-Ray Diffraction from Isolated and Strongly Aligned Gas-Phase Molecules with a FreeElectron Laser, Phys. Rev. Lett. 112, 083002 (2014).

[8] B. Erk et al., Imaging charge transfer in iodomethane upon X-ray photoabsorption, Science 345, 288 (2014).

[9] Z. Huang and K.-J. Kim, Review of x-ray free-electron laser theory, Phys. Rev. ST Accel. Beams 10, 034801 (2007).

[10] H. Ye, J. S. Kienitz, S. Fang, S. Trippel, M. E. Swanwick, P. D. Keathley, L. F. Velásquez-García, G. Cirmi, G. M. Rossi, A. Fallahi, O. D. Mücke, J. Küpper, and F. X. Kärtner, in Ultrafast Phenomena XIX, Springer Proceedings in Physics Vol. 162, edited by K. Yamanouchi, S. Cundiff, R. de Vivie-Riedle, M. Kuwata-Gonokami, and L. DiMauro (Springer International Publishing, Cham, Switzerland, 2015), p. 663.

[11] A. R. Bainbridge and W. A. Bryan, Velocity map imaging of femtosecond laser induced photoelectron emission from metal nanotips, New J. Phys. 16, 103031 (2014).

[12] H. Lee, S. Karkare, L. Cultrera, A. Kim, and I. V. Bazarov, Review and demonstration of ultra-low-emittance photocathode measurements, Rev. Sci. Instrum. 86, 073309 (2015).

[13] H. Ihee, V. A. Lobastov, U. M. Gomez, B. M. Goodson, R. Srinivasan, C. Y. Ruan, and A. H. Zewail, Direct imaging of transient molecular structures with ultrafast diffraction, Science 291, 458 (2001).

[14] B. J. Siwick, J. R. Dwyer, R. E. Jordan, and R. J. Dwayne Miller, An atomic-level view of melting using femtosecond electron diffraction, Science 302, 1382 (2003).

[15] M. Gulde, S. Schweda, G. Storeck, M. Maiti, H. K. Yu, A. M. Wodtke, S. Schäfer, and C. Ropers, Ultrafast low-energy electron diffraction in transmission resolves polymer/ graphene superstructure dynamics, Science 345, 200 (2014).
[16] M. Krüger, M. Schenk, and P. Hommelhoff, Attosecond control of electrons emitted from a nanoscale metal tip, Nature (London) 475, 78 (2011).

[17] G. Herink, D. R. Solli, M. Gulde, and C. Ropers, Fielddriven photoemission from nanostructures quenches the quiver motion, Nature (London) 483, 190 (2012).

[18] A. Mustonen, P. Beaud, E. Kirk, T. Feurer, and S. Tsujino, Five picocoulomb electron bunch generation by ultrafast laser-induced field emission from metallic nano-tip arrays, Appl. Phys. Lett. 99, 103504 (2011).

[19] W. P. Putnam, R. G. Hobbs, P. D. Keathley, K. K. Berggren, and F.X. Kärtner, Optical-field-controlled photoemission from plasmonic nanoparticles, Nat. Phys. 13, 335 (2017).

[20] S. Tsujino, P. D. Kanungo, M. Monshipouri, C. Lee, and R. J. D. Miller, Measurement of transverse emittance and coherence of double-gate field emitter array cathodes, Nat. Commun. 7, 13976 (2016).

[21] F.X. Kärtner et al., AXSIS: Exploring the frontiers in attosecond X-ray science, imaging and spectroscopy, Nucl. Instrum. Methods Phys. Res., Sect. A 829, 24 (2016).

[22] A. Polyakov, C. Senft, K. F. Thompson, J. Feng, S. Cabrini, P. J. Schuck, H. A. Padmore, S. J. Peppernick, and W. P. Hess, Plasmon-Enhanced Photocathode for High Brightness and High Repetition Rate X-Ray Sources, Phys. Rev. Lett. 110, 076802 (2013).

[23] R. K. Li, H. To, G. Andonian, J. Feng, A. Polyakov, C. M. Scoby, K. Thompson, W. Wan, H. A. Padmore, and P. Musumeci, Surface-Plasmon Resonance-Enhanced Multiphoton Emission of High-Brightness Electron Beams from a Nanostructured Copper Cathode, Phys. Rev. Lett. 110, 074801 (2013).

[24] C. N. Berglund and W. E. Spicer, Photoemission studies of copper and silver: Theory, Phys. Rev. 136, A1030 (1964).

[25] C. N. Berglund and W. E. Spicer, Photoemission studies of copper and silver: Experiment, Phys. Rev. 136, A1044 (1964).

[26] W. F. Krolikowski and W. E. Spicer, Photoemission studies of the noble metals. I. Copper, Phys. Rev. 185, 882 (1969).

[27] W. F. Krolikowski and W. E. Spicer, Photoemission studies of the noble metals. II. Gold, Phys. Rev. B 1, 478 (1970).

[28] A. T. J. B. Eppink and D. H. Parker, Velocity map imaging of ions and electrons using electrostatic lenses: Application in photoelectron and photofragment ion imaging of molecular oxygen, Rev. Sci. Instrum. 68, 3477 (1997).

[29] A. I. Chichinin, K. H. Gericke, S. Kauczok, and C. Maul, Imaging chemical reactions-3D velocity mapping, Int. Rev. Phys. Chem. 28, 607 (2009).

[30] M. Stei, J. von Vangerow, R. Otto, A. H. Kelkar, E. Carrascosa, T. Best, and R. Wester, High resolution spatial map imaging of a gaseous target, J. Chem. Phys. 138, 214201 (2013).

[31] G. Petite, P. Agostini, R. Trainham, E. Mevel, and P. Martin, Origin of the high-energy electron emission from metals under laser irradiation, Phys. Rev. B 45, 12210 (1992).

[32] N. L. M. Müller, S. Trippel, and K. Długołęcki, and J. Küpper, Electron gun for diffraction experiments on controlled molecules, J. Phys. B 48, 244001 (2015).

[33] Scientific Instrument Services, SIMION 8.1, http://simion .com (2011). 
[34] J. H. Bechtel, W. L. Smith, and N. Bloembergen, Fourphoton photoemission from tungsten, Opt. Commun. 13, 56 (1975).

[35] A. Damascelli, G. Gabetta, A. Lumachi, L. Fini, and F. Parmigiani, Multiphoton electron emission from $\mathrm{Cu}$ and $\mathrm{W}$ : An angle-resolved study, Phys. Rev. B 54, 6031 (1996).

[36] D. R. Lide, CRC Handbook of Chemistry and Physics, 84th ed. (CRC Press, Boca Raton, 2003).

[37] M. N. Polyanskiy, Refractive index database, https:// refractiveindex.info (24 July 2017).

[38] C. J. Dasch, One-dimensional tomography: A comparison of Abel, onion-peeling, and filtered backprojection methods, Appl. Opt. 31, 1146 (1992).

[39] D. H. Dowell and J. F. Schmerge, Quantum efficiency and thermal emittance of metal photocathodes, Phys. Rev. ST Accel. Beams 12, 074201 (2009).

[40] R. T. Poole, R. C. G. Leckey, J. G. Jenkin, and J. Liesegang, Photoelectron angular distribution from gold, J. Electron Spectrosc. Relat. Phenom. 1, 371 (1972).

[41] Z. Pei and C. N. Berglund, Angular distribution of photoemission from gold thin films, Jpn. J. Appl. Phys. 41, L52 (2002).

[42] W. S. Fann, R. Storz, H. W. K. Tom, and J. Bokor, Direct Measurement of Nonequilibrium Electron-Energy Distributions in Subpicosecond Laser-Heated Gold Films, Phys. Rev. Lett. 68, 2834 (1992).

[43] W. S. Fann, R. Storz, H. W. K. Tom, and J. Bokor, Electron thermalization in gold, Phys. Rev. B 46, 13592 (1992).

[44] M. Aeschlimann, C. A. Schmuttenmaer, H. E. ElsayedAli, R. J. D. Miller, J. Cao, Y. Gao, and D. A. Mantell,
Observation of surface enhanced multiphoton photoemission from metal surfaces in the short pulse limit, J. Chem. Phys. 102, 8606 (1995).

[45] F. Banfi, C. Giannetti, G. Ferrini, G. Galimberti, S. Pagliara, D. Fausti, and F. Parmigiani, Experimental Evidence of Above-Threshold Photoemission in Solids, Phys. Rev. Lett. 94, 037601 (2005).

[46] P. B. Corkum, Plasma Perspective on Strong-Field Multiphoton Ionization, Phys. Rev. Lett. 71, 1994 (1993).

[47] S. Karkare, L. Cultrera, Y.-W. Hwang, R. Merluzzi, and I. Bazarov, 2-D energy analyzer for low energy electrons, Rev. Sci. Instrum. 86, 033301 (2015).

[48] J. Feng, J. Nasiatka, W. Wan, T. Vecchione, and H. A. Padmore, A novel system for measurement of the transverse electron momentum distribution from photocathodes a novel system for measurement of the transverse electron momentum distribution from photocathodes, Rev. Sci. Instrum. 86, 015103 (2015).

[49] G. Sciaini and R. J. D. Miller, Femtosecond electron diffraction: Heralding the era of atomically resolved dynamics, Rep. Prog. Phys. 74, 096101 (2011).

[50] M. Hattass, T. Jahnke, S. Schössler, A. Czasch, M. Schöffler, L. Ph. H. Schmidt, B. Ulrich, O. Jagutzki, F. O. Schumann, C. Winkler, J. Kirschner, R. Dörner, and H. Schmidt-Böcking, Dynamics of two-electron photoemission from $\mathrm{Cu}(111)$, Phys. Rev. B 77, 165432 (2008).

[51] A. Evtukh, H. Hartnagel, O. Yilmazoglu, H. Mimura, and D. Pavlidis, Vacuum Nanoelectronic Devices: Novel Electron Sources and Applications (Wiley, New York, 2015). 